\title{
Floristic and ecological characterization of habitat types on an inselberg in Minas Gerais, southeastern Brazil
}

\author{
Luiza F. A. de Paula ${ }^{*}$, Nara F. O. Mota², Pedro L. Viana² and João R. Stehmann ${ }^{3}$
}

Received: November 21, 2016

Accepted: March 2, 2017

\begin{abstract}
Inselbergs are granitic or gneissic rock outcrops, distributed mainly in tropical and subtropical regions. They are considered terrestrial islands because of their strong spatial and ecological isolation, thus harboring a set of distinct plant communities that differ from the surrounding matrix. In Brazil, inselbergs scattered in the Atlantic Forest contain unusually high levels of plant species richness and endemism. This study aimed to inventory species of vascular plants and to describe the main habitat types found on an inselberg located in the state of Minas Gerais, in southeastern Brazil. A total of 89 species of vascular plants were recorded (belonging to 37 families), of which six were new to science. The richest family was Bromeliaceae (10 spp.), followed by Cyperaceae (seven spp.), Orchidaceae and Poaceae (six spp. each). Life forms were distributed in different proportions between habitats, which suggested distinct microenvironments on the inselberg. In general, habitats under similar environmental stress shared common species and life-form proportions. We argue that floristic inventories are still necessary for the development of conservation strategies and management of the unique vegetation on inselbergs in Brazil.
\end{abstract}

Keywords: endemism, granitic and gneissic rock outcrops, life forms, terrestrial islands, vascular plants

\section{Introduction}

Brazil has the most diverse flora of seed plants in the world (32,109 accepted native species), of which more than $50 \%$ are endemic to the country (BFG 2015). The Atlantic Forest domain contributes greatly to this high species richness, being internationally recognized as a center of biodiversity and endemism and considered a hotspot for conservation priorities (Myers et al. 2000; Mittermeier et al. 2004). What is frequently overlooked about the Atlantic Forest is that rock outcrops are not only widespread within this domain but also support large numbers of endemics (Safford \& Martinelli 2000; Scarano 2002; Porembski 2007). Recent data revealed that of the 995 species of angiosperms occurring on rock outcrops within the Atlantic Forest domain, 416 are endemic to these formations (Stehmann et al. 2009). Nevertheless, there are still large gaps in our knowledge about the flora of various mountain regions throughout the Brazilian territory (Merirelles et al. 1999; Safford 1999; Martinelli 2007).

The term "Inselberg" was coined by Bornhardt in 1900, from the German language with "insel" meaning island and "berg" meaning mountain (Barthlott \& Porembski 2000b), to characterize individual or groups of monolithic mountains, which appear abruptly in the landscape and which mainly consist of granitic or gneissic rocks (Porembski et al. 1997). They are considered terrestrial habitat islands because of their strong spatial and ecological isolation (Porembskiet al. 2000). Due to the

\footnotetext{
${ }^{1}$ Universität Rostock, Institut für Biowissenschaften, Allgemeine und Spezielle Botanik, Wismarsche Str. 44/45, D-18057 Rostock, Germany

${ }^{2}$ Coordenação de Botânica, Museu Paraense Emílio Goeldi, Av. Magalhães Barata, 376, São Braz, 66040-170, Belém, PA, Brazil

${ }^{3}$ Departamento de Botânica, Universidade Federal de Minas Gerais, P.O. Box 486, 30161-970, Belo Horizonte, MG, Brazil

* Corresponding author: luizafap@gmail.com
} 
prevailing ecological filters, including lack of soil and water, high exposure to UV radiation, high temperatures and constant winds (Porembski \& Barthlott 2000; Scarano 2002), the community composition of inselbergs are clearly distinguished from their surrounding matrices (Barthlott et al. 1993; Porembski 2007; de Paula et al. 2015). Thus, a set of typical plant communities and habitat types have been described for these rock outcrops over a broad geographical scale (Porembski et al. 2000).

There are three worldwide hotspots of inselberg plant diversity: southeastern Brazil, Madagascar and southwestern Australia (Porembski 2007). In the Brazilian territory, the largest concentration of inselberg is in the Northeast Region (Ab'Sáber 1967; Vieira et al. 2015), although a large concentration of these rock outcrops is also present in the Southeast Region, encompassing the states of Rio de Janeiro, Espírito Santo, southern Bahia and adjacent parts of Minas Gerais, where inselbergs exhibit high plant species richness and elevated rates of endemism (Porembski et al. 1998; Safford \& Martinelli 2000; Taylor \& Zappi 2004). This core area seems to form a particular phytogeographical region in the Atlantic Forest, and recently, based on the highly diverse flora of mat-forming Bromeliaceae occurring on inselbergs, was baptized as Sugar Loaf Land (de Paula et al. 2016). Moreover, beta diversity (i.e., the species turnover between individual outcrops) seems to be unusually high (Porembski 2007; de Paula et al. 2016). Remarkably, many floristic and ecological aspects of inselberg vegetation in Sugar Loaf Land are still not satisfactorily explored (Safford 1999; Barthlott \& Porembski 2000b).

The Northeast Region of Minas Gerais is dominated by inselbergs that are in need of scientific attention because there is a significant lacuna of biological investigations (Oliveira-Filho et al. 2005) and almost nothing has been done with regard to floristic inventories. The elevated richness of inselbergs in the area has been documented by previous studies (Taylor \& Zappi 2004; Martinelli 2007). Nevertheless, anthropogenic disturbance is steeply increasing, mainly due to historical agricultural activities on landscapes surrounding the rock outcrops (MMA 2006), and there are no conservation units in the area. Studies conducted in this region already reinforced, and even highlight, the need of enhancing efforts to inventory inselbergs (Martinelli 2007).

This study aimed to inventory species of vascular plants and to describe the main habitat types found on an inselberg located in Minas Gerais in southeastern Brazil. We tested if these habitat types were determined by specific plant communities, and expected to find higher floristic and life-form similarities in habitats under similar environmental conditions.

\section{Materials and methods}

\section{Study site}

This study was performed on an inselberg inserted in a peripheral region of the Atlantic Forest matrix, in the Mucuri
Valley, northeastern Minas Gerais, close to the Caatinga and Cerrado domains (Fig. 1). Inselbergs are typical landscape elements in the area, and they are mainly surrounded by lowland fragments of semi-deciduous seasonal forest (Veloso et al. 1991) and degraded vegetation modified by farming and grazing (Fig. 2A). The rock outcrop studied is located on private land ( $\left.17^{\circ} 5^{\prime} 09^{\prime \prime} \mathrm{S}, 41^{\circ} 15^{\prime} 44^{\prime \prime} \mathrm{W}\right)$ with elevations ranging from 306 to $676 \mathrm{~m}$ a. s. l. The climate at the study area is tropical wet with a dry season during winter (Aw), mean annual temperature is about $22.9^{\circ} \mathrm{C}$ and the annual rainfall is ca. $959 \mathrm{~mm}$ (INMET 1992; Alvares et al. 2013).

\section{Sampling}

The floristic survey consisted of the collection of fertile specimens and was conducted between January 2011 and December 2015, with a total of 12 expeditions to the studied inselberg, covering all the different seasons. Sampling was restricted to vascular plant species arranged in vegetation patches of many shapes and sizes (Caiafa \& Silva 2005) (Fig. 2 ). The area surveyed was approximately $20 \mathrm{ha}$. The botanical material was processed according to the usual techniques for vascular plants (Fidalgo \& Bononi 1989) and voucher specimens were deposited in the BHCB herbarium (acronym according to Thiers 2016). Taxonomic identification was accomplished by means of specialized taxonomic literature, herbarium data and, when necessary, by sending duplicate specimens to specialists. The circumscription adopted for angiosperm families are in accordance with APG IV (2016); for monilophytes and lycophytes we followed PPG I (2016). Spelling of the names, synonymy and authors follow Flora do Brasil 2020 (2016). The plants were photographed in the field and a color guide to the most representative species is available at http://fieldguides.fieldmuseum.org/sites/ default/files/rapid-color-guides-pdfs/378.pdf (de Paula et al. 2013).

Based on phytosociological analyses and physiognomic criteria, a set of typical habitat types of inselbergs can be distinguished (Porembski 2007). In this study, habitat types were categorized according to a classification established for granitic and gneissic rock outcrops (Barthlott et al. 1993; Porembski 2007). The following habitats were sampled: crevices, ephemeral flush vegetation, epilithic vegetation, monocot mats and shallow depressions. Species life forms were classified according to Raunkiaer (1934).

In December 2015, with the purpose of comparing species composition among habitats, 50-m long transects were set in five different areas on the studied inselberg. In total, 17 transects were established: four transects in four different areas, plus an additional transect in a fifth area. The areas selected varied in declivity and direction of slopes. In each transect, we surveyed up to five randomly chosen patches. In each patch, we determined species presence/ absence and checked for the occurrence of invasive species (sensu Moro et al. 2012). For some transects, it was not 


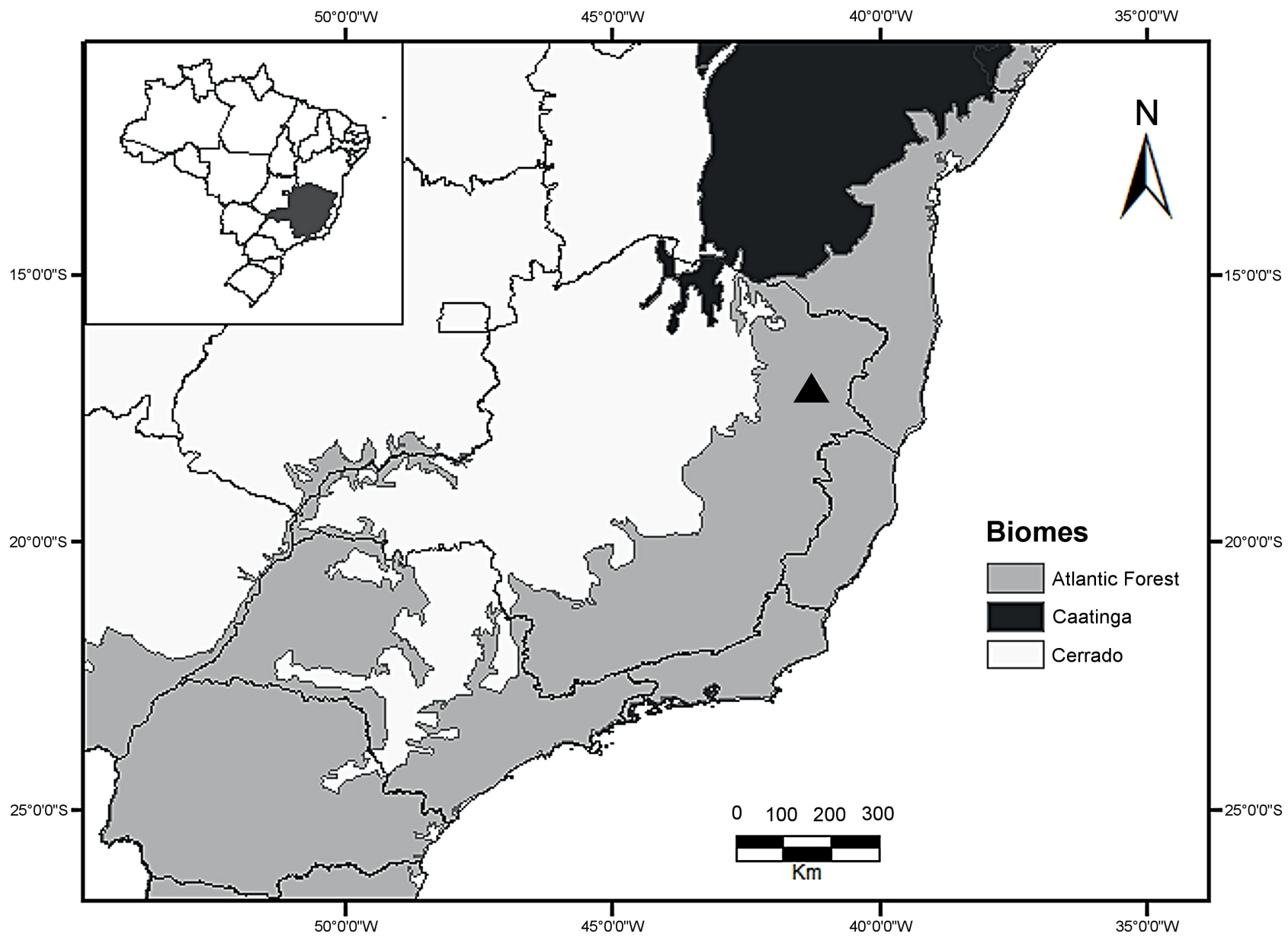

Figure 1. Location of the study area. The inselberg studied, represented by a triangle, is located in Minas Gerais State, southeastern Brazil. The rock outcrop is inserted in the Atlantic Forest matrix, yet close to the borders with Caatinga and Cerrado domains.
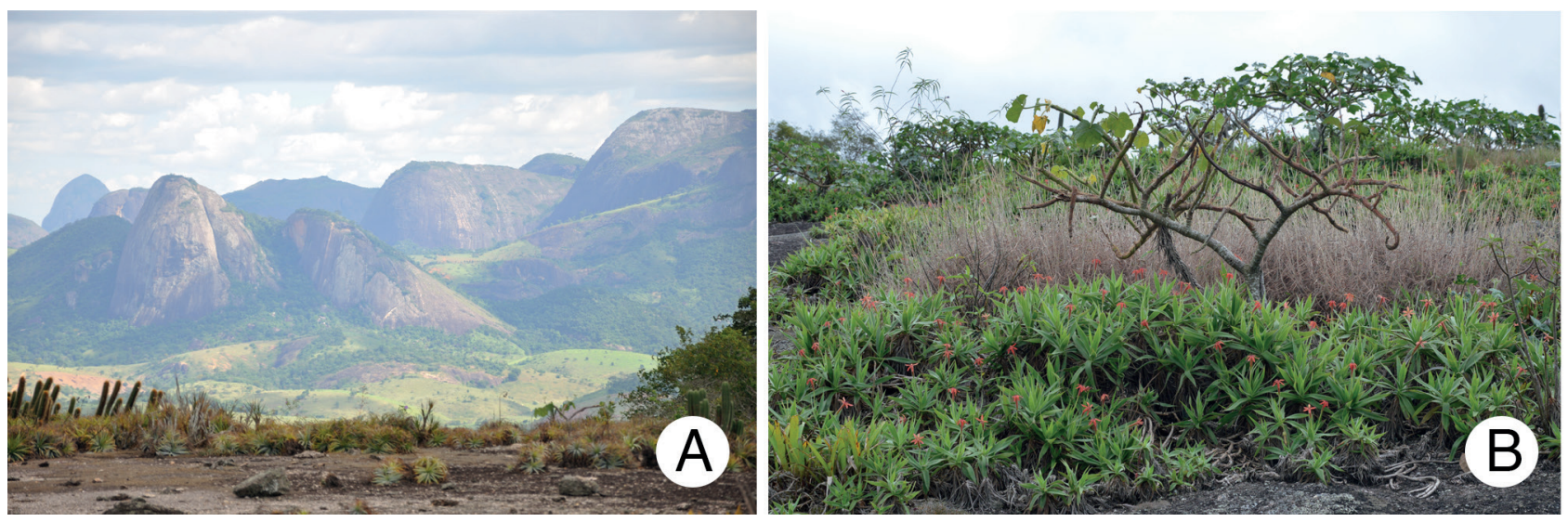

Figure 2. A. Overview of the inselberg studied in the Mucuri Valley, Minas Gerais State, southeastern Brazil. Vegetation patches are visible on the flat part of the rock, while in the background the landscape can be seen dominated by inselbergs. B. A typical vegetation patch: Barbacenia tomentosa forms a mat along the border; the shrub Cnidoscolus aff. lombardii occupies the central region; and the African invasive species Melinis repens is dominating the back part of the patch. Photo A by J.R. Stehmann, B by L.F.A. de Paula. 
possible to sample five patches due to the steepness of the surveyed areas. Altogether, we randomly selected 61 patches and categorized them as one of the above-mentioned habitats. Patch sampling reflected patch occurrence in the study area, therefore we sampled different numbers of patches of each category. Patch area ranged from 0.03 to $79.12 \mathrm{~m}^{2}(11.97 \pm 19.2$; SD $)$.

\section{Data analyses}

To compare plant species composition among the habitats sampled, we performed cluster analysis using the unweighted pair group method with arithmetic mean (UPGMA), based on a Sørensen similarity index. The calculation of the Sørensen similarity index and the UPGMA were both done with the software Past (Hammer et al. 2001). Species life forms were recorded for each habitat type and their proportions compared using Pearson's Chi-square test in $\mathrm{R}$ ( $\mathrm{R}$ Development Core Team 2015).

\section{Distribution, endemism and conservation}

Species distribution by phytogeographical domain and limitation to inselbergs were determined based on the literature (whenever possible original manuscripts of species descriptions were reviewed); online databases such as Flora do Brasil 2020 (JBRJ 2016a), Virtual Herbarium of Plants and Fungi (INCT 2016) and JBRJ-Jabot (JBRJ 2016b); and consultation with specialists. Information regarding threatened species was based on the Brazilian Official List (MMA 2014) and the Red Book of Brazilian Flora (Martinelli \& Moraes 2013).

\section{Results}

\section{Floristics}

A total of 89 species of vascular plants belonging to 37 families and 73 genera were found, consisting of 10 pteridophytes (three lycophytes and seven monilophytes) and 79 angiosperms (one magnoliid, 39 monocots and 39 eudicots) (Tab. 1). The family with the highest number of species was Bromeliaceae (10 spp.), followed by Cyperaceae (seven spp.), Orchidaceae and Poaceae (six spp. each), and Apocynaceae, Asteraceae and Velloziaceae (five spp. each). The richest genera were Mandevilla (Apocynaceae), Selaginella (Selaginellaceae), Tillandsia (Bromeliaceae) and Vellozia (Velloziaceae) (three spp. each).

Three species on the inselberg studied were described as new to science: Axonopus graniticola (Poaceae; Viana \& de Paula 2013), up to now known from inselbergs in the type locality in Minas Gerais and also in Espírito Santo; Bradea borrerioides (Rubiaceae; Oliveira \& Sobrado 2016), belonging to a genus restricted to the Atlantic Forest in
Minas Gerais, Rio de Janeiro and Espírito Santo; and the recently described Anthurium mucuri (Araceae; Gonçalves \& de Paula 2016). Three more species remain to be described, one belonging to Mandevilla (Apocynaceae; JF Morales unpubl. res.), one belonging to Pleroma (Melastomataceae; FS Meyer unpubl. res.), both also known from inselbergs of Espírito Santo, and another to Scleria (Cyperaceae; R Trevisan unpubl. res.), so far only found in the study area.

At least five new records for the state of Minas Gerais were recorded (Tab. 1): Begonia aguiabrancensis (Begoniaceae), Encyclia spiritusanctensis (Orchidaceae), Pitcairnia azouryi (Bromeliaceae), Sinningia aghensis (Gesneriaceae) and Schwenckia nova-veneciana (Solanaceae). These species were previously considered endemic to the state of Espírito Santo. In addition, three species were cited as rare for the Brazilian flora in Giulietti et al. (2009): Marsdenia otoniensis (Apocynaceae; up to now only known from the type material, collected more than 50 years ago), Stigmaphyllon crenatum (Malpighiaceae) and Tabebuia reticulata (Bignoniaceae).

\section{Habitat types}

Shallow depressions were the richest habitat on the studied inselberg, with 77 species recorded, of which 45 were exclusive to this habitat. This habitat consisted of depressions on rock, filled with shallow substrate and colonized by herbs, shrubs and trees, giving a heterogeneous aspect to the vegetation cover. On depressions with a thin layer of substrate it was common to find subshrubs like Begonia aguiabrancensis (Begoniaceae), Cololobus rupestris (Asteraceae), Cuphea sessilifolia (Lythraceae), Marsdenia otoniensis (Apocynaceae), monocots such as Axonopus graniticola (Poaceae), Anthurium mucuri, Philodendron edmundoi (Araceae), Cyrtopodium glutiniferum (Orchidaceae), Vellozia spp. (Velloziaceae) and some cacti, like Coleocephalocereus spp. and Pilosocereus brasilensis subsp. ruschianus. On the depressions with deeper substrate, shrubs were dominant, such as Cnidoscolus aff. lombardii, Croton nepetifolius, Stillingia argutedentata (Euphorbiaceae), Mandevilla spp. (Apocynaceae), Stachytarpheta gesnerioides var. gesnerioides (Verbenaceae), Pleroma spp. (Melastomataceae), and also trees were found: Erythroxylum nummularia (Erythroxylaceae), Tabebuia reticulata (Bignoniaceae) and Wunderlichia azulensis (Asteraceae).

Monocot mats were formed by dense stands of monocotyledons and occurred on both flat and inclined open rocky slopes; they have a mat-like appearance and are firmly attached to the rock by dense wiry roots. This habitat was composed of 15 species. The most typical mat-forming species were Alcantarea simplicisticha, Encholirium gracile, Pitcairnia azouryi, Portea petropolitana, Vriesea neoglutinosa, (Bromeliaceae), Trilepis lhotzkiana (Cyperaceae), Barbacenia spp. and Vellozia spp. (Velloziaceae). Non-monocot species were also recognized as mat-formers in the area, such as the members of the lycophyte genus Selaginella (Selaginellaceae), which also grows as "carpets" on rock. 
Table 1. List of lycophyte, monilophyte and angiosperm species recorded on an inselberg in Mucuri Valley, Minas Gerais State, southeastern Brazil. Phytogeographic Domains (sensu Flora do Brasil 2020): Am = Amazonia, $\mathrm{Ca}=\mathrm{Caatinga}, \mathrm{Ce}=\mathrm{Cerrado}, \mathrm{AF}=\mathrm{Atlantic}$ Forest, $\mathrm{Pm}=$ Pampa, $\mathrm{Pn}=$ Pantanal, All = all Domains. Habitat types: $\mathrm{C}=$ crevices, $\mathrm{EFV}=$ ephemeral flush vegetation, $\mathrm{EV}=$ epilithic vegetation, $\mathrm{MM}=$ monocot mat, $\mathrm{SD}=$ shallow depression. Life forms: $\mathrm{Ch}=$ chamephytes, $\mathrm{Cr}=\mathrm{cryptophyte}, \mathrm{Hc}=$ hemicryptophytes, $\mathrm{Ph}$ = phanerophayte, $\mathrm{Th}$ = therophyte. The following notations were also included: ! = endangered species (based on Martinelli \& Moraes 2013), ${ }^{*}=$ first record for Minas Gerais State. In bold, species endemic to inselbergs. Vouchers: L = L.F.A. de Paula and A = L.O. Azevedo

\begin{tabular}{|c|c|c|c|c|}
\hline $\begin{array}{l}\text { FAMILY - specialist } \\
\text { Species }\end{array}$ & $\begin{array}{l}\text { Habitat } \\
\text { type }\end{array}$ & $\begin{array}{l}\text { Life- } \\
\text { form }\end{array}$ & Voucher & $\begin{array}{l}\text { Phytogeografic } \\
\text { Domain }\end{array}$ \\
\hline \multicolumn{5}{|l|}{ SELAGINELLACEAE - T.E. Almeida \& A. Salino } \\
\hline Selaginella convoluta (Arn.) Spring & SD, MM & $\mathrm{Ch}$ & \multirow{2}{*}{$\begin{array}{l}\text { L } 97 \\
\text { L } 390\end{array}$} & $\mathrm{Ca}, \mathrm{Ce}, \mathrm{AF}$ \\
\hline S. jungermannioides (Gaudich.) Spring & $\mathrm{SD}, \mathrm{MM}$ & $\mathrm{Hc}$ & & $\mathrm{AF}$ \\
\hline S. sellowii Hieron. & SD,MM & Hc & L 306 & $\mathrm{Ca}, \mathrm{Ce}, \mathrm{AF}$ \\
\hline \multicolumn{5}{|l|}{ ANEMIACEAE - T.E. Almeida \& A. Salino } \\
\hline Anemia ferruginea Humb. \& Bonpl. ex Kunth & SD & $\mathrm{Hc}$ & L 147 & $\mathrm{Am}, \mathrm{Ca}, \mathrm{Ce}, \mathrm{AF}$ \\
\hline A. villosa Humb. \& Bonpl. ex Willd. & SD & $\mathrm{Hc}$ & L 133 & $\mathrm{Ce}, \mathrm{AF}$ \\
\hline \multicolumn{5}{|l|}{ BLECHNACEAE - T.E. Almeida \& A. Salino } \\
\hline Blechnum occidentale L. & SD & $\mathrm{Hc}$ & L 352 & $\mathrm{Am}, \mathrm{Ca}, \mathrm{Ce}, \mathrm{AF}$ \\
\hline \multicolumn{5}{|l|}{ POLYPODIACEAE - T.E. Almeida \& A. Salino } \\
\hline Microgramma vaccinifolia (Langsd. \& Fisch.) Copel. & SD & $\mathrm{Ph}$ & L 343 & $\mathrm{Ce}, \mathrm{AF}$ \\
\hline Serpocaulon latipes (Langsd. \& Fisch.) A.R.Sm. & SD & $\mathrm{Hc}$ & L 686 & $\mathrm{AF}$ \\
\hline \multicolumn{5}{|l|}{ PTERIDACEAE - T.E. Almeida \& A. Salino } \\
\hline Cheilanthes geraniifolia (Weath.) R.M.Tryon \& A.F.Tryon & $\mathrm{EV}, \mathrm{SD}$ & $\mathrm{Hc}$ & L 135 & $\mathrm{Ce}, \mathrm{AF}$ \\
\hline Doryopteris collina (Raddi) J.Sm. & $\mathrm{EV}, \mathrm{SD}$ & $\mathrm{Hc}$ & L 93 & $\mathrm{Am}, \mathrm{AF}$ \\
\hline \multicolumn{5}{|l|}{ MAGNOLIIDS } \\
\hline \multicolumn{5}{|l|}{ PIPERACEAE - D.L. Ambrosio } \\
\hline Peperomia incana (Haw.) Hook. & SD & $\mathrm{Ph}$ & L 139 & $\mathrm{AF}$ \\
\hline \multicolumn{5}{|l|}{ MONOCOTS } \\
\hline \multicolumn{5}{|l|}{ AMARYLLIDACEAE - J. Dutilh } \\
\hline Hippeastrum glaucescens (Mart.) Herb. & SD & $\mathrm{Cr}$ & L 636 & $\mathrm{Ca}, \mathrm{Ce}, \mathrm{AF}$ \\
\hline \multicolumn{5}{|l|}{ ARACEAE - E.G. Gonçalves } \\
\hline Anthurium mucuri E.G.Gonç. \& L.F.A. de Paula & $\mathrm{SD}, \mathrm{C}$ & $\mathrm{Hc}$ & L 136 & $\mathrm{AF}$ \\
\hline Philodendron edmundoi G.M. Barroso & $\mathrm{SD}, \mathrm{EV}$ & $\mathrm{Ch}$ & L 328 & $\mathrm{AF}$ \\
\hline \multicolumn{5}{|l|}{ BROMELIACEAE - L. Versieux, R.C. Forzza \& R. Louzada } \\
\hline Alcantarea simplicisticha Leme \& A.P.Fontana & $\mathrm{MM}, \mathrm{C}, \mathrm{SD}, \mathrm{EV}$ & $\mathrm{Hc}$ & L 87 & $\mathrm{AF}$ \\
\hline Encholirium gracile L.B.Sm. ! & $\mathrm{MM}, \mathrm{C}, \mathrm{SD}, \mathrm{EV}$ & $\mathrm{Ch}$ & L 234 & $\mathrm{AF}$ \\
\hline Orthophytum compactum L.B.Sm. & $\mathrm{SD}, \mathrm{C}, \mathrm{EV}$ & $\mathrm{Hc}$ & L 86 & $\mathrm{Ca}, \mathrm{Ce}, \mathrm{AF}$ \\
\hline Pitcairnia azouryi Martinelli \& Forzza * & MM & $\mathrm{Ch}$ & L 607 & $\mathrm{AF}$ \\
\hline Portea petropolitana (Wawra) Mez & MM & $\mathrm{Ch}$ & L 331 & $\mathrm{AF}$ \\
\hline Tillandsia gardneri Lindl. & SD & $\mathrm{Ph}$ & L 249 & $\mathrm{Ca}, \mathrm{Ce}, \mathrm{Af}, \mathrm{Pm}$ \\
\hline T. recurvata (L.) L. & SD & $\mathrm{Ph}$ & L 375 & $\mathrm{Ca}, \mathrm{Ce}, \mathrm{AF}, \mathrm{Pm}$ \\
\hline T. stricta Sol. & SD & $\mathrm{Ph}$ & L 326 & $\mathrm{Ca}, \mathrm{Ce}, \mathrm{AF}, \mathrm{Pm}$ \\
\hline Vriesea vellozicola Leme \& J.A.Siqueira & SD & $\mathrm{Hc}$ & L 639 & $\mathrm{AF}$ \\
\hline V. neoglutinosa $\mathrm{Mez}$ & $\mathrm{MM}, \mathrm{C}, \mathrm{SD}$ & $\mathrm{Hc}$ & L 124 & $\mathrm{AF}$ \\
\hline \multicolumn{5}{|l|}{ BURMANNIACEAE - M.O. Pivari } \\
\hline Burmannia capitata (Walter ex J.F.Gmel.) Mart. & EFV & Th & L 357 & $\mathrm{Am}, \mathrm{Ce}, \mathrm{AF}$ \\
\hline \multicolumn{5}{|l|}{ COMMELINACEAE } \\
\hline Tradescantia sp. & SD & $\mathrm{Ch}$ & L 154 & - \\
\hline \multicolumn{5}{|l|}{ CYPERACEAE - R. Trevisan } \\
\hline Bulbostylis lagoensis (Boeckeler) Prata \& M.G.López & SD, EFV & Th & L 690 & $\mathrm{Ce}, \mathrm{AF}$ \\
\hline Cyperus coriifolius Boeckeler & MM, SD & $\mathrm{Hc}$ & L 282 & $\mathrm{Ce}, \mathrm{AF}$ \\
\hline C. luzulae (L.) Retz & EFV & $\mathrm{Hc}$ & L 637 & All \\
\hline Fuirena umbellata Rottb. & EFV & Th & L 806 & All \\
\hline Rhynchospora tenuis Link & EFV & Th & L 807 & All \\
\hline Scleria sp. nov. & SD & $\mathrm{Hc}$ & L 653 & $\mathrm{AF}$ \\
\hline Trilepis lhotzkiana Nees ex Arn. & MM, C, SD, EV & $\mathrm{Ch}$ & L 134 & $\mathrm{Am}, \mathrm{Ca}, \mathrm{Ce}, \mathrm{AF}$ \\
\hline \multicolumn{5}{|l|}{ ORCHIDACEAE - P.L. Viana } \\
\hline Acianthera prolifera (Herb. ex Lindl.) Pridgeon \& M.W.Chase & $\mathrm{EV}, \mathrm{SD}$ & $\mathrm{Hc}$ & L 346 & $\mathrm{Ce}, \mathrm{AF}$ \\
\hline Cyrtopodium glutiniferum Raddi & SD & $\mathrm{Hc}$ & $\mathrm{L} 115$ & $\mathrm{AF}$ \\
\hline
\end{tabular}


Table 1. Cont.

\begin{tabular}{|c|c|c|c|c|}
\hline $\begin{array}{l}\text { FAMILY - specialist } \\
\text { Species }\end{array}$ & $\begin{array}{l}\text { Habitat } \\
\text { type }\end{array}$ & $\begin{array}{l}\text { Life- } \\
\text { form }\end{array}$ & Voucher & $\begin{array}{c}\text { Phytogeografic } \\
\text { Domain }\end{array}$ \\
\hline Encyclia spiritusanctensis L.C.Menezes * & SD & $\mathrm{Ch}$ & L 140 & $\mathrm{AF}$ \\
\hline Pseudolaelia geraensis Pabst & EV & $\mathrm{Ch}$ & L 338 & $\mathrm{AF}$ \\
\hline P. vellozicola (Hoene) C.Porto \& Brade & SD, EV & $\mathrm{Ch}$ & L 245 & $\mathrm{Ce}, \mathrm{AF}$ \\
\hline Prescottia montana Barb.Rodr. & SD & $\mathrm{Hc}$ & L 333 & $\mathrm{Ca}, \mathrm{Ce}, \mathrm{AF}$ \\
\hline \multicolumn{5}{|l|}{ POACEAE - P.L. Viana } \\
\hline Andropogon bicornis L. & EFV & $\mathrm{Hc}$ & L 298 & \\
\hline Axonopus graniticola Viana & SD & $\mathrm{Hc}$ & L 145 & \\
\hline Ichnanthus cf. dasycoleus Tutin & SD & Th & L 356 & \\
\hline Melinis repens (Willd.) Zizka & SD, EFV & $\mathrm{Ch}$ & L 395 & \\
\hline Paspalum cf. paniculatum L. & EFV & Hc & L 678 & \\
\hline Setaria sp. & SD & Th & L 673 & \\
\hline \multicolumn{5}{|l|}{ VELLOZIACEAE - R. Mello-Silva } \\
\hline Barbacenia purpurea Hook. & MM & $\mathrm{Ch}$ & L 640 & \\
\hline B. tomentosa Mart. & MM, SD, C & $\mathrm{Ch}$ & L 288 & \\
\hline Vellozia aff. hirsuta Goethart \& Henrard & MM, SD & $\mathrm{Ch}$ & L 314 & \\
\hline V. plicata Mart. & $\mathrm{MM}, \mathrm{C}, \mathrm{SD}, \mathrm{EV}$ & $\mathrm{Ph}$ & L 287 & \\
\hline V. pulchra L.B.Sm. ! & SD & $\mathrm{Ph}$ & L 90 & \\
\hline \multicolumn{5}{|l|}{ EUDICOTS } \\
\hline \multicolumn{5}{|l|}{ APOCYNACEAE - A. Rapini \& I. Koch } \\
\hline Ditassa cf. longicaulis (E.Fourn.) Rapini & SD & $\mathrm{Ph}$ & L 397 & $\mathrm{Ce}, \mathrm{AF}$ \\
\hline Mandevilla grazielae M.F.Sales et al. & SD, C & $\mathrm{Cr}$ & L 153 & $\mathrm{Ca}, \mathrm{AF}$ \\
\hline M. tenuifolia (J.C.Mikan) Woodson & SD & $\mathrm{Cr}$ & L 606 & $\mathrm{Am}, \mathrm{Ca}, \mathrm{Ce}, \mathrm{AF}$ \\
\hline Mandevilla sp. nov. & SD & $\mathrm{Cr}$ & L 611 & $\mathrm{AF}$ \\
\hline Marsdenia otoniensis Fontella \& Morillo! & SD & $\mathrm{Ph}$ & L 137 & $\mathrm{AF}$ \\
\hline \multicolumn{5}{|l|}{ ASTERACEAE - E.K.O. Hattori \& A.C. Fernandes } \\
\hline Baccharis salicifolia (Ruiz \& Pav.) Pers. & SD & $\mathrm{Ph}$ & L 645 & $\mathrm{Am}, \mathrm{Ce}, \mathrm{AF}$ \\
\hline Cololobus rupestris (Gardner) H.Rob. ! & SD, C & $\mathrm{Ph}$ & L 359 & $\mathrm{AF}$ \\
\hline Lepidaploa cotoneaster (Willd. ex Spreng.) H.Rob. & SD & $\mathrm{Ph}$ & L 248 & $\mathrm{Ce}, \mathrm{AF}$ \\
\hline Mikania microcephala DC. & SD & $\mathrm{Ph}$ & L 396 & $\mathrm{Ce}, \mathrm{AF}$ \\
\hline Wunderlichia azulensis Maguire \& G.M.Barroso! & SD, C & $\mathrm{Ph}$ & L 131 & $\mathrm{AF}$ \\
\hline \multicolumn{5}{|l|}{ BEGONIACEAE - L. Kollmann } \\
\hline Begonia aguiabrancensis L.Kollmann * & $\mathrm{SD}, \mathrm{C}$ & $\mathrm{Ph}$ & L 141 & $\mathrm{AF}$ \\
\hline \multicolumn{5}{|l|}{ BIGNONIACEAE } \\
\hline Tabebuia reticulata A.H.Gentry & SD, $C$ & $\mathrm{Ph}$ & L 401 & $\mathrm{Ca}, \mathrm{AF}$ \\
\hline \multicolumn{5}{|l|}{ CACTACEAE - D. Zappi } \\
\hline Coleocephalocereus buxbaumianus Buining! & SD, EV, C & $\mathrm{Ph}$ & L 320 & $\mathrm{AF}$ \\
\hline C. fluminensis (Miq.) Backeb.! & $\mathrm{SD}, \mathrm{EV}, \mathrm{C}$ & $\mathrm{Ph}$ & L 629 & $\mathrm{AF}$ \\
\hline Pilosocereus brasiliensis ssp. ruschianus (Buining \& Brederoo) Zappi ! & SD, EV & $\mathrm{Ph}$ & L 321 & $\mathrm{Ca}, \mathrm{AF}$ \\
\hline \multicolumn{5}{|l|}{ DIOSCOREACEAE - D. Araújo } \\
\hline Dioscorea polygonoides Humb. \& Bonpl. ex Willd. & SD & $\mathrm{Cr}$ & L 683 & $\mathrm{Ca}, \mathrm{AF}$ \\
\hline \multicolumn{5}{|l|}{ ERYTHROXYLACEAE } \\
\hline Erythroxylum nummularia Peyr & SD & $\mathrm{Ph}$ & L 91 & $\mathrm{Ca}, \mathrm{AF}$ \\
\hline \multicolumn{5}{|l|}{ EUPHORBIACEAE - R. Riina } \\
\hline Cnidoscolus aff. lombardii Fern.Casas & $\mathrm{SD}, \mathrm{C}$ & $\mathrm{Ph}$ & L 130 & $\mathrm{AF}$ \\
\hline Croton nepetifolius Baill. & SD & $\mathrm{Ph}$ & L 138 & $\mathrm{Ca}, \mathrm{AF}$ \\
\hline Stillingia argutedentata Jabl. & SD & $\mathrm{Ph}$ & L 132 & $\mathrm{AF}$ \\
\hline \multicolumn{5}{|l|}{ FABACEAE } \\
\hline Aeschynomene sp. & SD & $\mathrm{Ph}$ & L 144 & - \\
\hline \multicolumn{5}{|l|}{ GESNERIACEAE - A. Chautems \& M. Peixoto } \\
\hline Sinningia aghensis Chautems ! * & SD & $\mathrm{Cr}$ & L 642 & $\mathrm{AF}$ \\
\hline S. brasiliensis (Regel \& Schmidt) Wiehler \& Chataumes & SD & $\mathrm{Cr}$ & L 676 & $\mathrm{Ca}, \mathrm{AF}$ \\
\hline Paliavana prasinata (Ker Gawl.) Benth. & SD & $\mathrm{Ph}$ & L 283 & $\mathrm{AF}$ \\
\hline \multicolumn{5}{|l|}{ LENTIBULARIACEAE - M.O. Pivari } \\
\hline Utricularia subulata L. & EFV & Th & L 162 & $\mathrm{Am}, \mathrm{Ca}, \mathrm{Ce}, \mathrm{AF}$ \\
\hline
\end{tabular}


Table 1. Cont.

\begin{tabular}{|c|c|c|c|c|}
\hline $\begin{array}{c}\text { FAMILY - specialist } \\
\text { Species }\end{array}$ & $\begin{array}{l}\text { Habitat } \\
\text { type }\end{array}$ & $\begin{array}{l}\text { Life- } \\
\text { form }\end{array}$ & Voucher & $\begin{array}{c}\text { Phytogeografic } \\
\text { Domain }\end{array}$ \\
\hline \multicolumn{5}{|l|}{ LOASACEAE } \\
\hline Aosa parviflora (Schrad. ex DC.) Weigend & SD & Th & L 105 & $\mathrm{AF}$ \\
\hline \multicolumn{5}{|l|}{ LYTHRACEAE } \\
\hline Cuphea sessilifolia Mart. & SD & $\mathrm{Ph}$ & L 152 & $\mathrm{Ca}, \mathrm{Ce}, \mathrm{AF}$ \\
\hline \multicolumn{5}{|l|}{ MALPIGHIACEAE - A. Amorim } \\
\hline Stigmaphyllon crenatum C.E.Anderson! & SD & Hc & A 75 & $\mathrm{AF}$ \\
\hline \multicolumn{5}{|l|}{ MALVACEAE } \\
\hline Melochia cf. morongii Britton & SD & $\mathrm{Ph}$ & L 677 & $\mathrm{Ce}, \mathrm{AF}$ \\
\hline Pseudobombax cf. crassipes Ravenna & SD & $\mathrm{Ph}$ & L 378 & $\mathrm{Ce}, \mathrm{AF}$ \\
\hline \multicolumn{5}{|l|}{ MELASTOMATACEAE - P.J.F. Guimarães \& F.S. Meyer } \\
\hline Cambessedesia eichleri Cogn. & SD & $\mathrm{Ph}$ & L 241 & $\mathrm{Ce}, \mathrm{AF}$ \\
\hline Pleroma sp. nov. (D.Don) Cogn. & SD, C & $\mathrm{Ph}$ & L 168 & Ce, $\mathrm{AF}$ \\
\hline Pleroma sp. & SD & $\mathrm{Ph}$ & L 163 & - \\
\hline \multicolumn{5}{|l|}{ PLANTAGINACEAE } \\
\hline Achetaria crenata (Ronse \& Philcox) V.C.Souza & EFV, SD & $\mathrm{Ph}$ & L 157 & $\mathrm{Ca}, \mathrm{AF}$ \\
\hline \multicolumn{5}{|l|}{ PORTULACACEAE } \\
\hline Portulaca hirsutissima Cambess. & SD, EV, MM & $\mathrm{Ch}$ & L 394 & $\mathrm{Ca}, \mathrm{Ce}, \mathrm{AF}$ \\
\hline \multicolumn{5}{|l|}{ RUBIACEAE - R. Salas } \\
\hline Bradea borrerioides J.A. Oliveira \& Sobrado & SD & $\mathrm{Ph}$ & L 303 & $\mathrm{AF}$ \\
\hline \multicolumn{5}{|l|}{ SOLANACEAE - J.R. Stehmann } \\
\hline Schwenckia nova-veneciana Carvalho *! & SD & Th & L 239 & $\mathrm{AF}$ \\
\hline \multicolumn{5}{|l|}{ TALINACEAE } \\
\hline Talinum paniculatum (Jacq.) Gaertn. & SD & Th & L 178 & $\mathrm{Am}, \mathrm{Ca}, \mathrm{Ce}, \mathrm{AF}$ \\
\hline \multicolumn{5}{|l|}{ VERBENACEAE } \\
\hline Stachytarpheta gesnerioides var. gesnerioides Cham. & SD & $\mathrm{Ph}$ & L 166 & $\mathrm{Ce}, \mathrm{AF}$ \\
\hline
\end{tabular}

Although crevices were not very common on the studied inselberg, they were found to contain 17 species, but none of which were exclusive to this habitat. Shrubby species (Mandevilla grazielae, Cololobus rupestris, Cnidoscolus aff. lombardii), cacti (Coleocephalocereus spp.) and bromeliads (Alcantarea simplicisticha, Encholirium gracile, Orthophytum compactum) were the most common components recorded.

The exposed rock surface was covered by lichens and cyanobacteria, though some isolated vascular plants grew directly on the rock. Fifteen species were identified as epilithic, such as the orchids Acianthera prolifera, Pseudolaelia geraensis and P. vellozicola (Orchidaceae) - the latter also occurred epiphytically on Vellozia spp. -, Coleocephalocereus spp. (Cactaceae) and Philodendron edmundoi (Araceae).

Ephemeral flush vegetation formed on substrate that was wet due to the proximity of a natural pond (on the top of the inselberg) and an artificial dam. Here ten species were found, of which seven were exclusive. Annual species were preponderant, such as Burmanniaceae (Burmannia capitata), Cyperaceae (Cyperus luzulae, Bulbostylis lagoensis, Fuirena umbellata, Rynchospora tenuis), Lentibulariaceae (Utricularia subulata), Poaceae (Andropogon bicornis, Melinis repens, Paspalum paniculatum) and Plantaginaceae (Achetaria crenata).

From phytosociological sampling, 58 species of angiosperms were recorded (out of the 89 inventoried species). Of the 61 selected patches, 23 patches were categorized as shallow depressions, 22 as monocot mats, seven as ephemeral flush vegetation, five as epilithic vegetation and four as crevices. The UPGMA cluster analysis identified a cluster between monocot mats and crevices, and both were floristically similar to shallow depressions. Ephemeral flush vegetation was the most different habitat with regard to species composition. The cophenetic correlation was 0.94 (Fig. 3).

\section{Life forms}

The life-form spectrum indicated a predominance of phanerophytes (34 spp./38.20\%), followed by hemicryptophytes (23 spp./25.84\%) and chamaephytes (15 spp./16.85\%). Life forms were present in varying proportions among the habitats analyzed, with a predominance of phanerophytes $(44.7 \%)$ in shallow depressions, chamaephytes $(64.28 \%)$ in monocot mats, phanerophytes $(52.9 \%)$ in crevices, chamaephytes (40\%) in epilithic vegetation and therophytes (50\%) in ephemeral flush vegetation. The Pearson's Chi-square test indicated a strong statistically significantly interaction between life forms and habitat types $\left(\chi^{2}=51.537, P=\right.$ 0.000) (Fig. 4). 
The present study did not consider epiphytes and lianas in the biological spectrum in order to allow for comparisons with previous studies on inselbergs, which usually did not consider these two life-form categories. Therefore we report here as epiphytes the species Tillandsia gardneri, T. recurvata, $T$. stricta, Vriesea vellozicola (facultative), $V$. neoglutinosa (facultative) (Bromeliaceae); Pseudolaelia vellozicola (facultative) (Orchidaceae) and Microgramma vaccinifolia (Polypodiaceae); and as vines the species Ditassa cf. longicaulis, Mandevilla sp. nov. (Apocynaceae), Mikania microcephala (Asteraceae) and Dioscorea polygonoides (Dioscoreaceae).

\section{Endemism, distribution and conservation}

At least 27 species were considered potentially endemic to inselbergs (Tab. 1). We highlight the six new species and other species belonging to typical families on rock outcrops, such as Bromeliaceae: Alcantarea simplicisticha (Leme et al. 2008), Encholirium gracile (Martinelli \& Moraes 2013), Pitcairnia azouryi (Martinelli \& Forzza 2006), Vriesea vellozicola (Siqueira-Filho \& Leme 2006); Cactaceae: Coleocephalocereus buxbaumianus, C. fluminensis, Pilosocereus brasiliensis ssp. ruschianus (Taylor \& Zappi 2004); Orchidaceae: Encyclia spiritusanctensis (Menezes 1991), Pseudolaelia geraensis (Menini Neto et al. 2013); Apocynaceae: Mandevilla grazielae (Sales et al. 2006); and Velloziaceae: Barbacenia purpurea (Suguiyama et al. 2014), Vellozia pulchra (Smith \& Ayensu 1976).

The pattern of distribution of the inselberg flora showed that most of the species (63\%) were not exclusive to the Atlantic Forest domain. We observed that $48.3 \%$ of the species co-occurred in the Cerrado domain and $38.2 \%$ also in the Caatinga domain. None of the inventoried species were included in the Official List of Threatened Species of the Brazilian Flora (MMA 2014), but ten species were mentioned in the Red Book of the Brazilian Flora (Martinelli \& Morais 2013): Coleocephalocereus buxbaumianus, C. fluminensis, Cololobus rupestris, Encholirium gracile, Marsdenia otoniensis, Sinningia aghensis, Stigmaphyllon crenatum, Schwenckia novaveneciana, Vellozia pulchra and Wunderlichia azulensis.

With regard to biological invasion, the exotic African grass Melinis repens was recorded in the area, and it was considered to be the only invasive species there according to Moro et al. (2012). The proportion of invasive was null in patches categorized as epilithic vegetation, monocot mats and crevices. However, the invasive species $M$. repens could indeed establish in the ephemeral flush vegetation (14.3\% of the sampled patches) and dominate shallow depressions ( $30 \%$ of the sampled patches) (Fig. 2B). In the present survey, some native species found among the selected patches seemed not to be originally part of the inselberg flora, such as Andropogon bicornis, Baccharis salicifolia and Cyperus luzulae, however, we did not consider them in the proportion of invasive species.

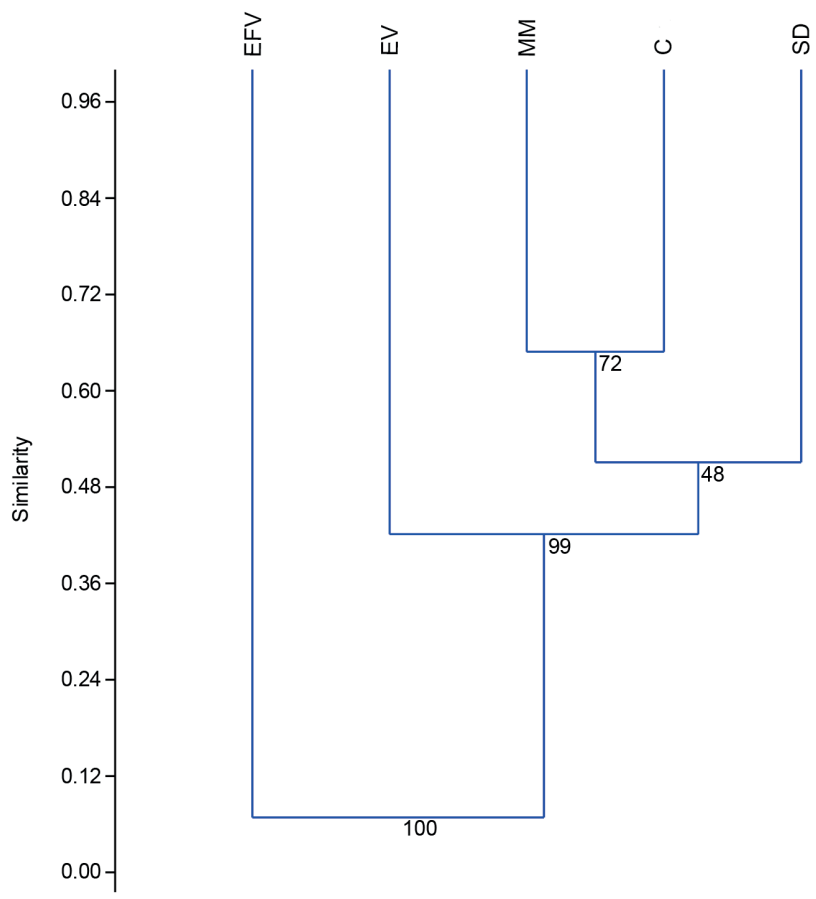

Figure 3. Cluster analysis, using the unweighted pair group method with arithmetic mean (UPGMA) and Sørensen similarity index, of the habitats found on an inselberg sampled in Minas Gerais State, southeastern Brazil, in relation to the presence of vascular plant species. Habitat abbreviations: EFV = ephemeral flush vegetation, $\mathrm{EV}=$ epilithic vegetation, $\mathrm{MM}=$ monocot mat $\mathrm{C}=$ crevice, $\mathrm{SD}=$ shallow depression. The cophenetic correlation was 0.94 .

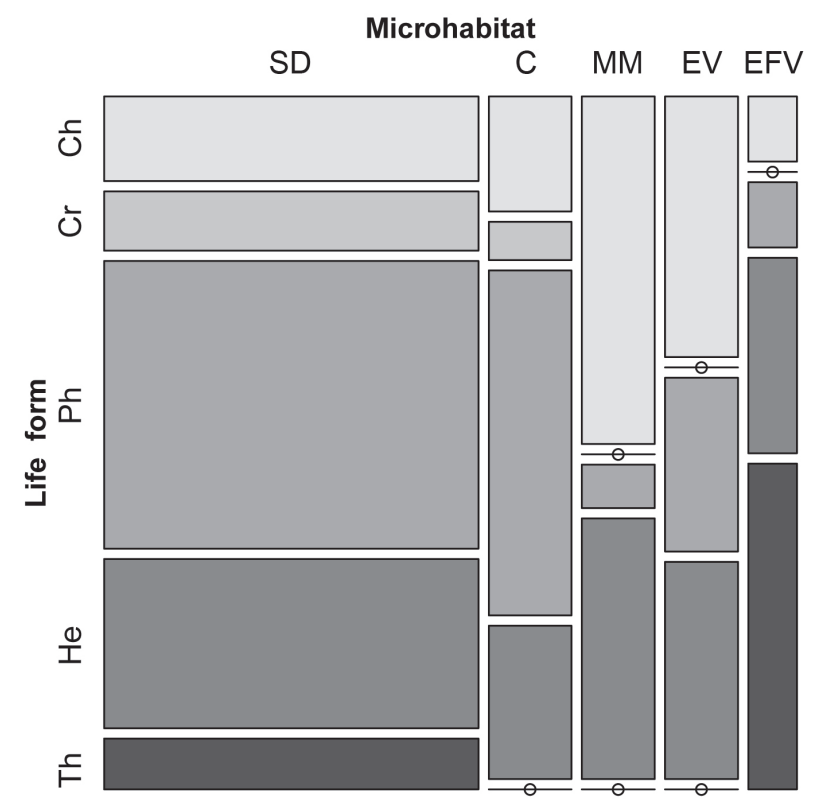

Figure 4. Percentage of life forms in each habitat record on an inselberg located in Minas Gerais State, southeastern Brazil. Life form abbreviations: $\mathrm{Ph}=$ phanerophyte, $\mathrm{Ch}=$ chamephyte, $\mathrm{Cr}=$ criptophyte, $\mathrm{H}=$ hemicryptophyte, $\mathrm{Th}=$ therophyte. Habitat abbreviations: $\mathrm{SD}=$ shallow depression, $\mathrm{MM}=$ monocot mat, $\mathrm{EFV}=$ ephemeral flush vegetation, $\mathrm{EV}=$ epilithic vegetation, $C=$ crevices. White circles cut by a line mean no occurrence of the respective life form in the corresponding habitat type. 


\section{Discussion}

The richest families recorded from the inselberg of the present study were also those well represented in other surveys of neotropical inselberg flora (Barthlott \& Porembski 2000a; Safford \& Martinelli 2000; Caiafa \& Silva 2005; Ribeiro et al. 2007; Gomes \& Alves 2009). Monocot families, such as Bromeliaceae, Orchidaceae, Cyperaceae and Velloziaceae, stood out among the flora of the studied inselberg, with many rupicolous and saxicolous members, which posses adaptive strategies that contribute to their survival on rock outcrops (Smith \& Ayensu 1976; Benzing 2000; Menini Neto et al. 2013). Habitat types were circunscribed by specific plant communities, however, there were greater shared species than expected. In general, habitats under similar environmental stress showed similar floristic and life-form composition. Furtermore, the high number of new species found on just one inselberg is noteworthy, and reflects the lack of floristic inventories in the area.

Within Bromeliaceae, typical genera of rock outcrops were found (e.g. Alcantarea, Encholirium, Orthophytum, Pitcairnia, Tillandsia and Vriesea). These genera dominate inselberg landscapes in Brazil (de Paula et al. 2016) and some have been the subject of studies of molecular and evolutionary biology (Barbará et al. 2007; Palma-Silva et al. 2011), which showed that intraspecific gene flow among inselbergs for certain bromeliads is unusually low (PalmaSilva et al. 2011), reinforcing the island-like characterization of these outcrops. It is important to note that mat-forming Bromeliaceae possess particular traits to cope with the drought conditions of inselbergs (de Paula et al. 2016). This is the case with xeromorphic species belonging to Encholirium, Orthophytum and Pitcairnia, for example, which possess succulent leaves, and the water-storage tank bromeliads, such as the species belonging to Alcantarea and Vriesea (Givnish et al. 2014).

In contrast to water-storage, many inselberg-specific plant species are desiccation-tolerant. This strategy has evolved in various clades of vascular plants, and are common among angiosperms and pteridophyte families on Brazilian inselbergs (Porembski \& Barthlott 2000). In our survey, this strategy has been seen in families such as Cyperaceae (Trilepis lhotzkiana) and Velloziaceae (e.g.Vellozia spp. and Barbacenia spp.), and within the pteridophyte genera Anemia, Cheilanthes, Doryopteris and Selaginella. All these genera have been described as desiccation-tolerant by previous studies (Porembski \& Barthlott 2000; Porembski 2007).

With regard to eudicots, the genus Mandevilla (Apocynaceae) stood out among the most specious genera found in this study. Species of this genus are often cited as typical elements of granitic outcrops, usually endemics (Porembski et al. 1998; Sales et al. 2006). Interestingly, Martins \& Alves (2008) identified anatomical features of Mandevilla spp., particularly in the leaves, that could be related to resistance against high temperature, insolation and water stress.

It is important to mention that a common phenomenon on inselbergs in southeastern Brazil is the widespread occurrence of species displaying a wide spectrum of morphological variation, which can cause extreme difficulties in their taxonomic treatment (Mello-Silva 2004; Versieux \& Wanderley 2010). Highly variable (polymorphic) species, whose variation is partly correlated with ecology and geography, are called ochlospecies (Cronk 1998). In our study, extensive intraspecific variation was found especially in populations of monocot taxa, such as Anthurium, Philodendron (Araceae), Alcantarea, Encholirium, Pitcairnia (Bromeliaceae), Barbacenia and Vellozia (Velloziaceae), which made the identification of these taxa even more difficult. Anthurium mucuri was cautiously recognized as a new species (Gonçalves \& de Paula 2013), after assessing other rock outcrops. Nevertheless, populations of taxa such as Encholirium and Vellozia are known to be highly polymorphic within populations of an inselberg and among populations of neighboring inselbergs, and thus need to be investigated further. The consideration of these species-complexes is important not only for solving taxonomic problems but also for understanding processes of the evolution of species on terrestrial islands, such as inselbergs.

Concerning the cluster analysis, crevices and monocot mats were floristically most similar, and both shared many common species with shallow depressions. The low support for the position of shallow depressions is probably due to its many exclusive species (such as shrubs and trees), which might have affected the position in the dendrogram. Furthermore, the habitat epilithic vegetation also had a considerable floristic relationship with crevices, monocot mats and shallow depressions (around $40 \%$ ). The great floristic similarities between these plant communities was a sign that these habitats are not completely isolated from each other; that is, most of the species occurring in crevices, epilithic vegetation and mats also occurred in shallow depressions. Therefore, the assumption made by Porembski et al. (2000) may need to be revaluated, since they called inselberg habitat types as "islands on islands", referring somehow to the floristic and ecological isolation of these habitats, which was not the case in the present study. However, the segregation of ephemeral flush vegetation from the other habitats was remarkable, with a number of specialists (such as Burmannia and Utricularia) related to seasonal humidity and lack of nutrients. Floristically, this habitat is, at the family and genus level, relatively uniform on inselbergs in tropical and temperate zones (Porembski 2007). Carnivorous plants (Lentibulariaceae), which are indicative of poor soils, usually are among the most prominent species of this community, as a consequence of low nutrient availability (Porembski et al. 2000). In this way, broader comparisons between habitat types located in different inselbergs should be performed, in order to 
elucidate if they represent different successional stages (Phillips 1981; Burbanck \& Phillips 1983) or if they are indeed experiencing different environmental filters (de Paula et al. 2015).

In our study, habitat types showed distinct proportions of life forms, and the relationship between these two variables was strongly significant. Since the distribution of life forms changes with environmental conditions (Raunkiaer 1934), it is likely that distinct microenvironments occur on the rock outcrop. Therophytes prevailed only on ephemeral flush vegetation, where annual species, including grasses and sedges, were preponderant. In previous studies, therophytes were also the main life form reported for this habitat (Porembski 2000b; Krieger et al. 2003). In resource-limited environments, such as epilithic vegetation and monocot mats, chamaephytes were more common. In addition, crevices and shallow depressions showed the predominance of phanerophytes, which may be due to the more favorable conditions in these habitats, such as greater amount of substrate, which allows the establishment of shrubby species.

Concerning the life-form spectrum for the entire inselberg community studied, phanerophytes comprised the largest proportion. Some studies of rock outcrops in Brazil found phanerophytes and/or hemicryptophytes to be predominant (Safford \& Martinelli 2000; Caiafa \& Silva 2005; Ribeiro et al. 2007; Gomes \& Alves 2010), while others exhibited a greater proportion of chamaephytes (Porembski et al. 1998; Conceição \& Giulietti 2002; Conceição et al. 2007) and terophytes (Gomes \& Sobral-Leite 2013). The large number of phanerophytes found in the present study may be due to the geomorphology of the studied inselberg, which is comprised of many depressions. This habitat allows the accumulation of larger amounts of substrate, enabling the establishment of woody species from the surrounding vegetation. Until now, a clear pattern regarding life-form spectrums of rock outcrops in the Atlantic Forest has not been recognized (Ribeiro et al. 2007). This absence of a pattern could be attributed to the fact that studies concerned with life-form spectrums of plant communities use different sources of data, such as presence/absence species lists or plant abundance, which may culminate in differing results. Hence, the only tendency observed is that the flora of inselbergs in tropical rainforests, such as the Atlantic Forest, contain a low percentage of therophytes (Safford \& Martinelli 2000; Caiafa \& Silva 2005; Ribeiro et al. 2007), whereas inselbergs in drier areas of northeastern Brazil (Gomes \& Alves 2010; Gomes \& Sobral-Leite 2013) and in West Africa (Porembski 2000b) are dominated by annual species.

Another outstanding result of this study was the high number of inselberg endemic species. Despite the lack of detailed knowledge about Brazilian inselbergs, and using the few inventories currently available, it can be seen that they possess high floristic diversity with regard to both number of species and endemics (Porembski 2007; Porembski et al. 1997; Martinelli 2007; de Paula et al. 2016), and it seems comparable only to inselbergs in Madagascar (Porembski 2007; Rabarimanarivo et al. 2017). An interesting fact is that West African inselbergs exhibit a low percentage of endemics, relative the inselbergs of Brazil and Madagascar (Porembski et al. 1997). Moreover, the high proportion of species shared between the studied inselberg flora and other phytogeographical domains, such as Caatinga and Cerrado, is notable. These domains are also subjected to harsh environmental conditions, and the mosaic vegetation of both comprises xerophytic plants adapted to intense dry seasons (Sampaio 1995; Ratter et al. 1997; Leal et al. 2005). It is possible to infer that inselbergs in this area represent discontinuous and scattered stepping stones for a xeric vegetation, which has already been observed in other tropical parts of the world (Burke 2002; Gröger \& Huber 2007).

Regarding biological invasions, inselberg environmental heterogeneity implies differences in invadeability, as there were some habitats more prone to invasion than others. In habitats with relatively greater resource availability and favorable characteristics for invasions, such as shallow depressions, Melinis repens was found to occur in large clumps. This species and other weeds have been found on several inselbergs throughout southeastern Brazil, and are considered a serious threat to these plant communities (Porembski et al. 1998; Porembski 2000a; de Paula et al. 2015). The critical point is that, when disturbance regimes are altered, rock outcrops become extremely vulnerable to invasion by non-native exotic organisms (Hooper 2009). Inselberg plant communities, as well as other types of outcrop vegetation (Jacobi et al. 2007), have minimal abilities to persist and recover, especially because many of their species possess slow growth rates and low dispersibility (Hooper 2009). Consequently, the opportunities for the establishment of invasive organisms increases, and repair and restoration become significantly more challenging for this type of vegetation (Hopper 2009).

Thus, in the context of increasing extraction of natural resources in Brazil (Ferreira et al. 2014), the rapid fragmentation of the Brazilian Atlantic Forest (Tabarelli et al. 2004) and steeply increasing threats to inselbergs, such as quarrying, mining and unsustainable extraction of attractive species of the flora (Martinelli 2007; Porembski et al. 2016), the refugia for vegetation on these outcrops may be jeopardized (Porembski et al. 2000). Additionally, this is valid not just for endemics, but also for species with broad distributions, since currently they are not found in the matrix, but instead on inselbergs (Pessoa \& Alves 2014). In some regions, such as northeastern Minas Gerais, these rock outcrops harbor the last remnants of forest fragments. In this context, we also highlight the fact that none of the species of the inventoried inselberg were present in the 
Official List of Threatened Species of the Brazilian Flora (MMA 2014), but were indicated as threatened in other sources such as the Red Book of Brazilian Flora (Martinelli \& Moraes 2013). Thus, the absence of threatened species in official lists can result in negative impacts by public conservation policies, and consequently compromise the conservation of the inselberg flora.

Due to the difficulty of access and the fact that many inselbergs are located in areas of grassland or forest fragments, the botanical community has overlooked the great potential of these outcrops. With regard to conservation, every remnant of native vegetation of rocky outcrops, no matter how small, is worth retaining, and should be investigated and preserved (Hooper 2009). Remnants can house populations of some organisms showing unexpected persistence (e.g. old lineages and old individuals, like some members of Velloziaceae) and enhanced resilience (Hopper 2009), new species and endemics, despite the massive fragmentation and loss of the surrounding vegetation.

The results presented here revealed new species, new records for the state of Minas Gerais, high plant species richness and elevated endemism on a single inselberg, which illustrated the uniqueness of Sugar Loaf Land (sensu de Paula et al. 2016). We argue that floristic inventories and research on the ecological determinants of plant species diversity on these rock outcrops are urgently needed, which will help in the management and conservation of this particular flora.

\section{Acknowledgements}

We thank de Paula and Addiny families, L. Azevedo, R. Fernandes, T. Mota, P. Burkowski, T. Jorge, F. Leite, M. Augsten, D. Negreiros, F. Silveira and S. Porembski for logistical support, help in field work and statistical analyses. We also thank the specialists who helped with plant identification and the grants from Conselho Nacional de Pesquisa e Desenvolvimento - CNPq (130469/2013-0; 309304/2013-0). This study was in partial fulfilment of the bachelor and master requirements of L. de Paula in Plant Biology.

\section{References}

Ab’Sáber AN. 1967. Domínios morfoclimáticos e províncias fitogeográficas do Brasil. Revista Orientação 3: 45-48.

Alvares CA, Stape JL, Sentelhas PC, Gonçalves JLM, Sparovek G. 2013. Köppen's climate classification map for Brazil. Meteorologische Zeitschrift 22: 711-728.

APG IV - The Angiosperm Phylogeny Group. 2016. An update of the Angiosperm Phylogeny Group classification for the orders and families of flowering plants: APG IV. Botanical Journal of the Linnaean Society 181: 1-20.

Barbará T, Martinelli G, Fay MF, Mayo SJ, Lexer C . 2007. Population differentiation and species cohesion in two closely related plants adapted to neotropical high altitude 'inselbergs', Alcantarea imperialis and Alcantarea geniculata (Bromeliaceae). Molecular Ecology 16: 19811992.
Barthlott W, Gröger A, Porembski S. 1993. Some remarks on the vegetation of tropical inselbergs: diversity and ecological differentiation. Compte rendu des Séances de la Société de Biogéographie 69: 105-124.

Barthlott W, Porembski S. 2000a. Vascular plants on inselbergs: systematic overview. In: Porembski S, Barthlott W. (eds.) Inselbergs - biotic diversity of isolated rock outcrops in tropical and temperate regions. Berlin, Springer-Verlag. p. 103-116.

Barthlott W, Porembski S. 2000b. Why Study Inselbergs?. In: Porembski S, Barthlott W. (eds.) Inselbergs - biotic diversity of isolated rock outcrops in tropical and temperate regions. Berlin, Springer-Verlag. p. 1-6.

Benzing DH. 2000. Bromeliaceae: profile of an adaptive radiation. Cambridge, Cambridge University Press.

BFG - The Brazil Flora Group. 2015. Growing knowledge: an overview of Seed Plant diversity in Brazil. Rodriguésia 66: 1085-1113.

Burbanck MP, Phillips DL. 1983. Evidence of plant succession on granite outcrops of the Georgia Piedmont. The American Midland Naturalist 109: 94-104.

Burke A. 2002. Are Namibian inselbergs conservation islands? A floral perspective: research in action. South African Journal of Science 98: 560.

Caiafa AN, Silva AF. 2005. Composição florística e espectro biológico de um campo de altitude no Parque Estadual da Serra do Brigadeiro, Minas Gerais-Brasil. Rodriguésia 56: 163-173.

Conceição AA, Giulietti AM. 2002. Floristic composition and structural aspects of campo rupestre on two plateaus in Pai Inacio Hill, Chapada Diamantina, Bahia, Brazil. Hoehnea 29: 37-48.

Conceição AA, Pirani JR, Meirelles ST. 2007. Floristics, structure and soil of insular vegetation in four quartzite-sandstone outcrops of "Chapada Diamantina", Northeast Brazil. Brazilian Journal of Botany 30: 641-656.

Cronk QCB. 1998. The ochlospecies concept. In: Huxley CR, Lock JM, Cutler DF. (eds.) Chorology, taxonomy and ecology of the African and Madagascan floras. Kew, Royal Botanic Gardens. p. 155-170.

de Paula LFA, Viana PL, Mota N, Augsten M, Leite F, Stehmann JR. 2013. Saxicolous Plants of an Inselberg in the Mucuri Valley. Chicago, Rapid Color Guides, The Field Museum. http://fieldguides.fieldmuseum. org/sites/default/files/rapid-color-guides-pdfs/378.pdf. 1 Apr. 2016.

de Paula LFA, Negreiros D, Azevedo LO, Fernandes RL, Stehmann JR, Silveira FAO. 2015. Functional ecology as a missing link for conservation of a resource-limited flora in the Atlantic forest. Biodiversity and Conservation 24: 2239-2253.

de Paula LFA, Forzza RC, Neri AV, Bueno ML, Porembski S. 2016. Sugar Loaf Land in south-eastern Brazil: a centre of diversity for matforming bromeliads on inselbergs. Botanical Journal of the Linnean Society 181: 459-476.

Ferreira J, Aragão LEOC, Barlow J, et al. 2014. Brazil's environmental leadership at risk. Science 346: 706-707.

Fidalgo O, Bononi VLR. 1989. Técnicas de coleta, preservação e herborização de material botânico. São Paulo, Governo do Estado de São Paulo, Secretaria do Meio Ambiente, Instituto de Botânica.

Giulietti AM, Rapini A, Andrade MJG, Queiroz LP, Silva JMC. 2009. Plantas raras do Brasil. Belo Horizonte, Conservação Internacional.

Givnish TJ, Barfuss MHJ, Ee B, et al. 2014. Adaptive radiation, correlated and con- tingent evolution, and net species diversification in Bromeliaceae. Molecular Phylogenetics and Evolution 71: 55-78.

Gomes P, Alves M. 2009. Floristic and vegetational aspects of an inselberg in the semi-arid region of Northeast Brazil. Edinburgh Journal of Botany 66: 329-346.

Gomes P, Alves M. 2010. Floristic diversity of two crystalline rocky outcrops in the Brazilian northeast semi-arid region. Brazilian Journal of Botany 33: 661-676.

Gomes P, Sobral-Leite M. 2013. Crystalline rock outcrops in the Atlantic Forest of northeastern Brazil: vascular flora, biological spectrum, and invasive species. Brazilian Journal of Botany 36: 111-123.

Gonçalves EG, de Paula LFA. 2016. A new species of Anthurium (Araceae) from Minas Gerais State, Southeastern Brazil. Phytotaxa 247: 281-286.

Gröger A, Huber O. 2007. Rock outcrop habitats in the Venezuelan Guayana lowlands: their main vegetation types and floristic components. Brazilian Journal of Botany 30: 599-609. 
Hammer Ø, Harper DAT, Ryan PD. 2001. PAST: Paleontological statistics software package for education and data analysis. Palaeontologia Electronica 4: 9.

Hopper SD. 2009. OCBIL theory: towards an integrated understanding of the evolution, ecology and conservation of biodiversity on old, climatically buffered, infertile landscapes. Plant and Soil 322: 49-86.

INCT - Institutos Nacionais de Ciência e Tecnologia. 2016. Virtual Herbarium of Plants and Fungi. http://inct.florabrasil.net/en/ herbario-virtual/. 1 Apr. 2016.

INMET - Instituto Nacional de Meteorologia. 1992. Normais climatológicas (1961-1990). Brasília, INMET.

Jacobi CM, Carmo FF, Vincent RC, Stehmann JR. 2007. Plant communities on ironstone outcrops: a diverse and endangered Brazilian ecosystem. Biodiversity and Conservation 16: 2185-2200.

JBRJ - Instituto de Pesquisas Jardim Botânico do Rio de Janeiro. 2016a. Flora do Brasil 2020 em construção. Rio de Janeiro, http:// floradobrasil.jbrj.gov.br. 1Apr. 2016.

JBRJ - Instituto de Pesquisas Jardim Botânico do Rio de Janeiro. 2016b. Jabot - Banco de dados da flora brasileira. http://www.jbrj.gov.br/ jabot. 1 Apr. 2016.

Krieger A, Porembski S, Barthlott W. 2003. Temporal dynamics of an ephemeral plant community: species turnover in seasonal rock pools on Ivorian inselbergs. Plant Ecology 167: 283-292.

Leal IR, Silva JMC, Tabarelli M, Lacher TE. 2005. Changing the course of biodiversity conservation in the Caatinga of northeastern Brazil. Conservation Biology 19: 701-706.

Leme EMC, Fraga CN, Kollmann LJC, Fontana AP. 2008. Three new Alcantarea species from Espírito Santo and Minas Gerais, Brazil. Journal of the Bromeliad Society 58: 205-216.

Martinelli G. 2007. Mountain biodiversity in Brazil. Brazilian Journal of Botany 30: 587-597.

Martinelli G, Forzza RC. 2006. Pitcairnia L'Hér.(Bromeliaceae): a new species, P. azouryi Martinelli \& Forzza, and notes on P. encholirioides LB Sm. Brazilian Journal of Botany 29: 603-607.

Martinelli G, Moraes MA. 2013. Red Book of Brazilian Flora. Jardim Botânico do Rio de Janeiro. http://cncflora.jbrj.gov.br/portal/pt-br/ livro. 1 Apr. 2016).

Martins S, Alves M. 2008. Aspectos anatômicos de espécies simpátridas de Mandevilla (Apocynaceae) ocorrentes em inselbergues de Pernambuco-Brasil. Rodriguésia 59: 369-380.

Mello-Silva R. 2004. Sistemática de Vellozia candida (Velloziaceae). Rodriguesia 55: 59-64.

Menezes LC. 1991. A large and showy new species from Brazil from northern Espirito Santo, Encyclia spiritusanctensis LC Men. Orchid Digest 55: 23.

Meirelles ST, Pivello VR, Joly CA. 1999. The vegetation of granite rock outcrops in Rio de Janeiro, Brazil, and the need for its protection. Environmental Conservation 26: 10-20.

Menini Neto L, Forzza RC, Berg C. 2013. Taxonomic revision of Pseudolaelia Porto \& Brade (Laeliinae, Orchidaceae). Acta Botanica Brasilica 27: 418-435.

Mittermeier RA, Gil PR, Hoffmann M, et al. 2004. Hotspots Revisited. Earth's biologically richest and most endangered terrestrial ecorregions. Mexico, CEMEX.

MMA - Ministério do Meio Ambiente. 2006. Projeto de Conservação e Utilização Sustentável da Diversidade Biológica Brasileira- PROBIO. Biodiversidade e conservação nos Vales dos Rios Jequitinhonha e Mucuri. Brasília, http://sistemas.mma.gov.br/sigepro/pub/display_ bibliografias.php?\%20vId_Gerenciamento $=1001 \&$ Destaque $=7.1$ Apr. 2016.

MMA - Ministério do Meio Ambiente. 2014. Lista Nacional Oficial de Espécies da Flora Ameaçadas de Extinção. Portaria 443, de 17 de dezembro de 2014. Brasília, MMA.

Moro MF, Souza VC, Oliveira-Filho AT, et al. 2012. Alienígenas na sala: o que fazer com espécies exóticas em trabalhos de taxonomia, florística e fitossociologia?. Acta Botanica Brasilica 26: 991-999.

Myers N, Mittermeier RA, Mittermeier CG, da Fonseca GAB, Kent J. 2000. Biodiversity hotspots for conservation priorities. Nature 403: 853-858.

Oliveira JA, Sobrado SV. 2016. Bradea borrerioides (Rubiaceae), a new species from Brazilian inselbergs. Phytotaxa 243: 83-90.
Oliveira-Filho AT, Tameirão-Neto E, Carvalho WA, et al. 2005. Análise florística do compartimento arbóreo de áreas de Floresta Atlântica sensu lato na região das Bacias do Leste (Bahia, Minas Gerais, Espírito Santo e Rio de Janeiro). Rodriguésia 56: 185-235.

Palma-Silva C, Wendt T, Pinheiro F, et al. 2011. Sympatric bromeliad species (Pitcairnia spp.) facilitate tests of mechanisms involved in species cohesion and reproductive isolation in Neotropical inselbergs. Molecular Ecology 20: 3185-3201.

Pessoa E, Alves M. 2014. Orchidaceae em afloramentos rochosos do estado de Pernambuco, Brasil. Rodriguésia 65: 717-734.

Phillips DL. 1981. Succession in granite outcrop shrub-tree communities. The American Midland Naturalist 106: 313-317.

Porembski S. 2000a. The invasibility of tropical granite outcrops ('inselbergs') by exotic weeds. Journal of the Royal Society of Western Australia 83: 131-137.

Porembski S. 2000b. West African inselberg vegetation. In: Porembski S, Barthlott W. (eds.) Inselbergs - biotic diversity of isolated rock outcrops in tropical and temperate regions. Berlin, Springer-Verlag. p. 177-211.

Porembski S. 2007. Tropical inselbergs: habitat types, adaptive strategies and diversity patterns. Brazilian Journal of Botany 30: 579-586.

Porembski S, Barthlott W. 2000. Granitic and gneissic outcrops (inselbergs) as centers of diversity for desiccation-tolerant vascular plants. Plant Ecology 151: 19-28.

Porembski S, Becker U, Seine R. 2000. Islands on islands: habitats on inselbergs. In: Porembski S, Barthlott W. (eds.) Inselbergs - biotic diversity of isolated rock outcrops in tropical and temperate regions. Berlin, Springer-Verlag. p. 49-67.

Porembski S, Martinelli G, Ohlemüller R, Barthlott W. 1998. Diversity and ecology of saxicolous vegetation mats on inselbergs in the Brazilian Atlantic rainforest. Diversity and Distributions 4: 107-119.

Porembski S, Seine R, Barthlott W. 1997. Inselberg vegetation and the biodiversity of granite outcrops. Journal of the Royal Society of Western Australia 80: 193-199.

Porembski S, Silveira FA, Fiedler PL, et al. 2016. Worldwide destruction of inselbergs and related rock outcrops threatens a unique ecosystem. Biodiversity and Conservation 25: 2827-2830.

PPG - Pteridophyte Phylogeny Group I. 2016. A community-derived classification for extant lycophytes and ferns. Journal of Systematics and Evolution 54: 563-603.

R Development Core Team. 2015. R: a language and environment for statistical computing. Vienna, R Foundation for Statistical Computing.

Rabarimanarivo MN, Porembski S, Phillipson PB, Ramandimbisoa B, Rakotoarivelo N, Andriambololonera S. 2017. The extraordinary botanical diversity of Malagasy inselbergs. Candollea (in press).

Ratter JA, Ribeiro JF, Bridgewater S. 1997. The Brazilian cerrado vegetation and threats to its biodiversity. Annals of Botany 80: 223-230.

Raunkiaer C. 1934. The life forms of plants and statistical geography. Oxford, Clarendon.

Ribeiro KT, Medina BMO, Scarano FR. 2007. Species composition and biogeographic relations of the rock outcrop flora on the high plateau of Itatiaia, SE-Brazil. Brazilian Journal of Botany 30: 623-639.

Safford HD. 1999. Brazilian Paramos I. An introduction to the physical environment and vegetation of the campos de altitude. Journal of Biogeography 26: 693-712.

Safford HD Martinelli G. 2000. Southeast Brazil. In: Porembski S, Barthlott W. (eds.) Inselbergs - biotic diversity of isolated rock outcrops in tropical and temperate regions. Berlin, Springer-Verlag. p 339-389.

Sales MF, Kinoshita LS, Simões AO. 2006. Eight new species of Mandevilla Lindley (Apocynaceae, Apocynoideae) from Brazil. Novon: A Journal for Botanical Nomenclature 16: 112-128.

Sampaio EVS. 1995. Overview of the Brazilian Caatinga. In: Bullock SH, Mooney HA, Medina E. (eds.) Seasonally dry tropical forests. Cambridge, Cambridge University Press. p. 35-63.

Scarano FR. 2002. Structure, function and floristic relationships of plant communities in stressful habitats marginal to the Brazilian Atlantic rainforest. Annals of Botany 90: 517-524.

Siqueira-Filho JA, Leme EM. 2006. Fragmentos de Mata Atlântica do nordeste: biodiversidade, conservação e suas bromélias. Rio de Janeiro, Andrea Jackobsson Estúdio Editorial Ltda. 
Smith LB, Ayensu ES. 1976. A revison of American Velloziaceae. Smithsonian Contributions to Botany 30: 1-172.

Stehmann JR, Forzza RC, Salino A, Sobral M, Costa DP, Kamino LHY. 2009. Plantas da Floresta Atlântica. Jardim Botânico do Rio de Janeiro, Rio de Janeiro.

Suguiyama VF, Silva EA, Meirelles ST, Centeno DC, Braga MR. 2014 Leaf metabolite profile of the Brazilian resurrection plant Barbacenia purpurea Hook.(Velloziaceae) shows two time-dependent responses during desiccation and recovering. Frontiers in Plant Science 96: $1-13$.

Tabarelli M, Silva MJC, Gascon C. 2004. Forest fragmentation, synergisms and the impoverishment of neotropical forests. Biodiversity and Conservation 13: 1419-1425.

Taylor NP, Zappi DC. 2004. Cacti of eastern Brazil. Kew, Royal Botanic Gardens.
Thiers B. 2016 [continuously updated]. Index Herbariorum: a global directory of public herbaria and associated staff. New York Botanical Garden's Virtual Herbarium. http://sweetgum.nybg.org/ih/. 1 Apr. 2016.

Veloso HP, Rangel Filho ALR, Lima JCA. 1991. Classificação da vegetação brasileira, adaptada a um sistema universal. Rio de Janeiro, Fundação Instituto Brasileiro de Geografia e Estatística.

Versieux L, Wanderley MGL. 2010. Delimitation of the Alcantarea extensa complex (Bromeliaceae) and a new species from Espírito Santo, Brazil. Rodriguésia 61: 421-429.

Viana PL, de Paula LFA. 2013. Axonopus graniticola, a new species of A. ser. Suffulti (Poaceae, Panicoideae, Paspaleae) from Minas Gerais, Brazil. Phytokeys 21: 7-16.

Vieira BC, Salgado AAR, Santos LJC. 2015. Landscapes and Landforms of Brazil - World Geomorphological Landscapes. Dordrecht, Springer. 